\title{
L'appréciation des rendements en fromagerie
}

\author{
par \\ J.-L. MAUBOIS* et G. MOCQUOT**
}

La " prédétermination " des rendements en fromagerie, c'est-àdire la prévision (avant la fabrication proprement dite) du poids de fromage qui peut être obtenu à partir d'une quantité déterminée de lait, devient de plus en plus nécessaire en raison de l'évolution de la technologie fromagère. Sans cela en effet, on ne peut concevoir la mécanisation ou même l'automatisation des différents traitements amenant le lait de l'état liquide à l'état de fromage prêt à affiner.

Or une telle prédétermination du rendement n'a jusqu'ici été envisagée qu'à partir des équations expérimentales du type $R=f$ (TB, TP) résultant de l'étude statistique de fabrications nombreuses bien contrôlées (Mocquot, Ricordeau et Auriol, 1963 ; Ricordeau et Mocquot, 1967 ; Portmann, Pierre et Vedrenne, 1968 ; Maubois, Ricordeau et Mocquot, 1970).

Si cette méthode donne d'excellents résultats (prévision de 93 p. 100 des variations du rendement (Maubois, Ricordeau et Mocquot, 1970)), elle présente le défaut de nécessiter chaque fois une expérimentation. Les différents coefficients des paramètres pris en considération dans les équations de prédétermination ne s'appliquent, en effet, qu'à une fabrication donnée, obtenue selon une technologie donnée.

Nous avons donc cherché à établir une prévision du rendement fromager plus générale, qui s'adapterait à tous les types de fromage et qui serait indépendante de la technologie utilisée pour la fabrication. Dans ce but, nous avons assimilé la transformation du lait en fromage à l'essorage (plus ou moins poussé selon le type de fromage) d'une éponge imbibée d'un liquide : la trame de l'éponge serait formée par la paracaséine (fixant la graisse) le liquide d'imbibition par le lactosérum, cette assimilation a déjà été utilisée précédemment (Maubois et Mocquot, 1967 ; Maubois, Mocquot et BlancPatin, 1967).

Ce « modèle de l'éponge » permet de calculer « le rendement fromager maximum " c'est-à-dire la quantité maximale de fromage de composition définie susceptible d'être obtenue à partir d'un lait de composition définie.

* Laboratoire de Recherches de Technologie Laitière I.N.R.A., 65, rue de SaintBrieuc (35) Rennes.

** Station Centrale de Recherches Laitières I.V.R.A., C.N.R.Z. (78) Jouy-en-Josas. 
Dans cette étude, nous nous sommes attachés, d'une part, à établir les modalités de calcul du « rendement fromager maximum » et d'autre part, à montrer comment on pouvait utiliser sur le plan pratique les éléments de ce calcul pour une prédétermination des rendements.

La paracaséine apportée par le lait mis en œuvre se retrouve dans le fromage obtenu avec ce lait. Soit, si l'on désigne la teneur en paracaséine* $^{*}$ du lait mis en œuvre par le symbole (M.A.C.), on peut écrire :

$\mathrm{R}_{\mathrm{s}} \cdot \mathrm{x} \cdot 10=$ (M.A.C.) $\cdot 100$

$\mathrm{R}_{\mathrm{M}}$ étant le poids de fromage obtenu avec $100 \mathrm{~kg}$ de lait.

$\mathrm{x}$ étant la quantité de paracaséine, exprimée en $\mathrm{g}$, contenue dans $100 \mathrm{~g}$ de fromage.

(M.A.C.) étant la teneur en paracaséine du lait exprimée en g pour $1000 \mathrm{~g}$ de lait.

L'équation conduit à :

$$
\mathrm{R}_{\mathrm{M}}=\frac{\text { (M.A.C.) } \cdot 100}{\mathrm{x} \cdot 10}
$$

Le rendement fromager maximum $\left(\mathrm{R}_{\mathrm{M}}\right)$ est donc égal au rapport des teneurs en paracaséine du lait mis en œuvre et du fromage obtenu avec ce lait.

La teneur en paracaséine du lait est facile à déterminer (Ricordeau et Mocquot, 1967 ; Maubois, Ricordeau et Mocquot, 1970). En outre la proportion de paracaséine dans les matières azotées totales varie peu. Par exemple, la proportion de la teneur en M.A.C. dans les matières azotées totales est de 74 p. 100 en moyenne pour le lait de vache cru et elle atteint 74,5 p. 100 en moyenne quand le lait est porté à $74^{\circ} \mathrm{C}$ pendant $20 \mathrm{~s}$ (Maubois, 1968).

La quantité de paracaséine contenue dans $100 \mathrm{~g}$ de fromage est égale à la différence entre la teneur en substance sèche dégraissée totale de ce fromage et la teneur en substance sèche dégraissée provenant du lactosérum de ce même fromage :

si (F) représente la quantité de substance sèche totale contenue dans $100 \mathrm{~g}$ de fromage,

si (g) représente la quantité de matière grasse contenue dans $100 \mathrm{~g}$ de fromage,

si (a) représente la quantité de substance sèche de lactosérum contenue dans $100 \mathrm{~g}$ de fromage,

on peut écrire :

$\mathrm{x}=\mathrm{F}-\mathrm{g}-\mathrm{a}$

* On emploie également les expressions suivantes :

- matières azotées coagulables par la présure,

- substance sèche insoluble. 
Dans une précédente étude (Maubois, Mocquot et Blanc-Patin, 1967) nous avons montré que si l'on appelle :

(y) la quantité d'eau libre contenue dans $100 \mathrm{~g}$ de fromage,

(N) la quantité d'eau liée au phosphoparacaséinate de calcium contenue dans $100 \mathrm{~g}$ de fromage,

(s) la teneur en substance sèche du lactosérum inclus dans le fromage exprimée en $\mathrm{g}$ pour $100 \mathrm{~g}$ de lactosérum, on peut écrire, d'une part :

$100-(\mathrm{F}+\mathrm{N})=\mathrm{y}$

et d'autre part :

$$
\begin{aligned}
& 2 \mathrm{~N}+\mathrm{y} \cdot \frac{\mathrm{s}}{100-\mathrm{s}}=\mathrm{F} \\
& \text { paracaséine }+ \text { substance sèche }=\text { substance sèche de } \\
& \text { du lactosérum fromage correspon- }
\end{aligned}
$$

Mais l'équation (5) s'appliquait à un fromage ne contenant pas de matière grasse, dans le cas d'un fromage qui en contient ( $g$ ), cette équation devient :

$$
2 \mathrm{~N}+\mathrm{g}+\mathrm{y} \cdot \frac{\mathrm{s}}{100-\mathrm{s}}=\mathrm{F}
$$

La résolution des équations 4 et 5 ' conduit à :

$$
\mathrm{y} \cdot \frac{\mathrm{s}}{100-\mathrm{s}}=\frac{200-3 \mathrm{~F}+\mathrm{g}}{200-\mathrm{s}} \cdot \mathrm{s}
$$

En remplaçant $\mathrm{x}$ par sa valeur dans l'équation (2), on obtient l'expression suivante du rendement fromager maximum :

$$
R_{\mathrm{M}}=\frac{[(\text { M.A.C. }) \text { 10] }(200-\mathrm{s})}{200(\mathrm{~F} \cdot \mathrm{g})-2 \mathrm{~s}(100-\mathrm{F})}
$$

Ainsi, la composition du fromage étant définie (teneur en graisse ; teneur en eau ; teneur en substance sèche du lactosérum inclus) la seule connaissance du taux de paracaséine du lait mis en œuvre suffit pour estimer le poids maximum de fromage qui peut être obtenu avec ce lait.

Une objection que l'on peut avancer est :

a) qu'il n'existe pas de méthode pratique de dosage de la teneur en substance sèche du lactosérum inclus dans le fromage et

b) -que cette teneur en substance sèche varie selon le lait mis en œuvre.

Ceci est exact mais : en première approximation on peut estimer que a) la teneur en substance sèche du lactosérum de fin d'égouttage est très voisine de la teneur en substance sèche du lactosérum inclus dans le fromage et b) les résultats obtenus avec 
des laits de grand mélange (Maubois, Ricordeau, Mocquot, 1970) montrent la faible variabilité (coefficient de variation à peine supérieur à 1 p. 100) de la teneur en substance sèche du lactosérum.

Dans la pratique industrielle courante et notamment en fromagerie de pâtes molles, on préfère exprimer le rendement du lait en fromage, non en kg de fromages obtenus à partir de $100 \mathrm{~kg}$ de lait, mais en nombre de litres ou de kilogs utilisés pour la fabrication d'un seul fromage. Dans l'exemple chiffré qui suit, nous avons indiqué de quelle manière il était possible d'utiliser les données ci-dessus pour déterminer le nombre de kilogs de lait à mettre en œuvre par unité de fromage.

Soit, par exemple, un fromage " étalon " du type Camembert possédant la composition suivante :

Poids frais

Substance sèche totale

Graisse pour $100 \mathrm{~g}$ de substance sèche totale

Substance sèche totale pour $100 \mathrm{~g}$ de fromage

Chlorure de sodium ajouté pour $100 \mathrm{~g}$ de fromage

$\begin{array}{rr}285,7 & \mathrm{~g} \\ 120 & \mathrm{~g} \\ 46 & \mathrm{~g} \\ 42 & \mathrm{~g} \\ 2 & \mathrm{~g}\end{array}$

La quantité de paracaséine contenue dans $100 \mathrm{~g}$ de fromage est égale à :

$$
\begin{aligned}
& \underset{\text { graisse }}{120-(55,2 \mathrm{~g}}+\underset{\text { sel ajouté }}{5,7 \mathrm{~g}}+\underset{\text { substance sèche }}{9,7 \mathrm{~g})}+\begin{array}{c}
49,4 \mathrm{~g} \\
\text { paracaséine contenue }
\end{array} \\
& \text { de lactosérum dans le fromage }
\end{aligned}
$$

La valeur de la substance sèche de lactosérum contenue dans le fromage, déterminée ci-dessus, a été obtenue en utilisant l'équation [6] et en supposant que la teneur en substance sèche du lactosérum inclus dans le fromage était égale à $7 \mathrm{~g} \mathrm{p} .100 \mathrm{~g}$.

Pour un lait de fabrication dont la teneur en protéines est égale à $32 \mathrm{~g}$ par $\mathrm{kg}$ le nombre de $\mathrm{kg}$ de lait à mettre en œuvre sera de :

$$
\frac{49,4}{32 \times 0,74}=2,09 \mathrm{~kg}
$$

La teneur en matière grasse du lait de fabrication sera :

$$
55,2 / 2,09=26,4 \mathrm{~g} / \mathrm{kg}
$$

Les chiffres ainsi obtenus devront être majorés, selon les caractéristiques propres à chaque usine, pour tenir compte des pertes en caillé et en graisse.

Dans une prochaine étude, nous montrerons, à partir de résultats obtenus en fabrication industrielle de fromage Camembert, la corrélation observée entre le rendement fromager maximum et le rendement réel observé en fabrication. 


\section{$R$ és u m é}

Le «modèle de l'éponge » proposé précédemment pour la transformation du lait en fromage, permet de calculer d'une manière relativement simple le "rendement fromager maximum » c'est-à-dire la quantité maximale de fromage de composition définie qui peut être obtenue à partir d'un lait de composition également définie. L'utilisation de la formule proposée, dans la pratique industrielle courante, est illustrée par un exemple chiffré de fabrication de fromage du type Camembert.

\section{Su $\mathrm{m}$ m a ry}

Assuming that cheese is similar to a sponge soaked in whey, one can estimate, for a well defined type of cheese, the maximum cheese yielding capacity of milk. The use of such a formula in standard industrial practice is given for Camembert cheesemaking.

\section{Références bibliographiques}

[1] Maubois (J. L.) et Mocouot (G.) (1967). - Comment ramener à la même teneur en substance sèche des fabrications de fromage en vue de comparer les « rendements" respectifs du lait en fromage. Rev. lait. franç., $239,15-18$.

[2] Maubois (J. L.), Mocouot (G.) et Blanc-Patin (E.) (1967). - Influence de la concentration du lait sur la quantité de substance sèche de "fromage " obtenue après coagulation de lait par la présure. Ann. Technol. agric., 16 (4), 287-299.

[3] Maubois (J. L.), Ricordeau (G.) et Mocouot (G.) (1970). - Etude des rendements en fromagerie de Camembert et de Saint-Paulin. Le Lait, 497, 351-373.

[4] Maubois (J. L.) (1968). - Résultats non publiés.

[5] Mocouot (G.), Blanc-Patin (E.), Sainclivier (M.), Rousseaux (P.) et Jeunet (R.) (1963). - Relation entre la teneur du lait en matière azotée et le poids de fromage obtenu. Ind. Lait., 203, 269-276.

[6] Portmann (A.), Pierre (A.) et Vedrenne (P.) (1968). - Relation entre teneurs en matière grasse et azotée du lait de chèvre et rendement en fromage. Rev. Lait. franç., 251, 97-101.

[7] Ricordeau (G.), Mocouot (G.) (1967). - Influence des variations saisonnières de la composition du lait de chèvre sur le rendement en fromage. Conséquences pratiques pour la sélection. Ann. Zootech., 16 (2), 165-181.

Reçu pour publication en avril 1971. 